\title{
Dimensional Reduction, Extended Topological Field Theories and Orbifoldization
}

\author{
Lukas Müller $^{a}$ and Lukas Woike ${ }^{b}$ \\ ${ }^{a}$ Max-Planck-Institut für Mathematik \\ Vivatsgasse 7, D-53111 Bonn \\ lmueller4@mpim-bonn.mpg.de \\ ${ }^{b}$ Fachbereich Mathematik \\ Universität Hamburg \\ Bereich Algebra und Zahlentheorie \\ Bundesstraße 55, D-20146 Hamburg \\ lukas. jannik. woike@uni-hamburg. de
}

\begin{abstract}
We prove a decomposition formula for the dimensional reduction of an extended topological field theory that arises as an orbifold of an equivariant topological field theory. Our decomposition formula can be expressed in terms of a categorification of the integral with respect to groupoid cardinality. The application of our result to topological field theories of Dijkgraaf-Witten type proves a recent conjecture of Qiu-Wang.
\end{abstract}

\section{Contents}

$\begin{array}{lll}1 & \text { Introduction } & 1\end{array}$

2 Equivariant field theories and their orbifolds 3

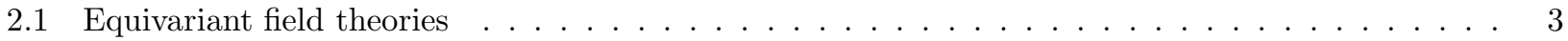

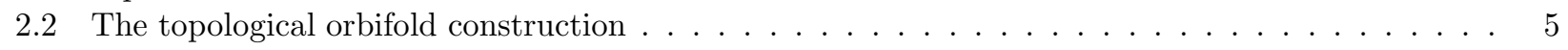

3 Dimensional reduction of equivariant topological field theories $\quad 7$

4 Application to Dijkgraaf-Witten theories 9

\section{Introduction}

This short note is concerned with the application of recently developed orbifoldization techniques for extended topological field theories to the subject of dimensional reduction. More concretely, we prove a decomposition formula for the dimensional reduction of a topological orbifold theory.

Topological field theories form a class of (quantum) field theories that can be mathematically axiomatized as symmetric monoidal functors out of the cobordism category [At88. For their relevance in physics, we refer e.g. to Kap10 and to [RW18 more specifically for applications in quantum computing.

Additionally, topological field theories have attracted interest in pure mathematics thanks to the manifold invariants they provide [RT91, TV92] and the deep connections between three-dimensional topological field theory and representation theory [Tur10, BDSPV15] that allow us to use low-dimensional topology for the study of algebraic objects like modular categories. Moreover, extended topological field theories and the cobordism 
hypothesis have become one of the driving forces behind many important developments in the theory of higher categories [Lur09].

Topological field theories come in different flavors. In particular, there are numerous interesting and wellstudied types of topological field theories featuring decorated cobordisms. In the monograph [Tur10-G], this decoration is chosen to be a map to some topological space, the target space. When the target space is the classifying space $B G$ of a (finite) group $G$, this leads to $G$-equivariant topological field theories. Constructions of classes of examples are available in TV12, MNS12, TV14, MW20. Of course, the decoration with maps to $B G$ amounts to a decoration with $G$-bundles. In this note, we will be concerned with once-extended (in the sequel, just 'extended' for brevity) $G$-equivariant topological field theories which are formally described as symmetric monoidal functors $Z: G$ - $\operatorname{Cob}_{n} \longrightarrow 2$ Vect from the symmetric monoidal oriented $G$-cobordism bicategory $G$-Cob ${ }_{n}$ to the symmetric monoidal bicategory of Kapranov-Voevodsky 2-vector spaces (these will always be over the complex numbers in this note). As in Tur10-G], these functors additionally have to satisfy a homotopy invariance property.

In the papers [SW19, SW18, SW20, a topological orbifold construction has been developed. For a given finite group $G$, this construction assigns to an extended $G$-equivariant topological field theory of any dimension a non-equivariant topological field theory of the same dimension, i.e. it takes the form of a functor

$$
-/ G: G \text {-TFT } \longrightarrow \text { TFT }, \quad Z \longmapsto Z / G .
$$

The image of a $G$-equivariant topological field theory $Z$ under this functor is called its orbifold theory and denoted by $Z / G$. The construction combines the computation of homotopy fixed points with the integration over essentially finite groupoids. When restricted to the circle in the three-dimensional case, the topological orbifold construction amounts to the categorical orbifoldization [Müg04, Kir04, GNN09] thereby opening a profitable topological perspective on this important algebraic operation.

The purpose of this note is to give a decomposition formula for the dimensional reduction of such an orbifold theory $Z / G$. For a fixed closed oriented manifold $T$ of dimension $r$, the dimensional reduction on $T$ (hereafter also referred to as $T$-reduction) assigns to an $n+r$-dimensional extended topological field theory $E$ an $n$ dimensional extended topological field theory $\operatorname{Red}_{T} E$ by evaluation of $E$ on manifolds of the form $M \times T$, where $M$ is of dimension $n, n-1$ and $n-2$, i.e. $\operatorname{Red}_{T} E=E(-\times T)$.

In order to formulate our main result, we extend the notion of dimensional reduction to equivariant topological field theories such that for a given $n+r$-dimensional $G$-equivariant topological field theory $Z: G$-Cob - $_{n+r} \longrightarrow$ 2 Vect, the $T$-reduction can be seen to produce a family of equivariant topological field theory indexed by all $G$-bundles over $T$. The family member $\left(\operatorname{Red}_{T} Z\right)^{P}$ belonging to a $G$-bundle $P \longrightarrow T$ is an $\operatorname{Aut}(P)$-equivariant topological field theory of dimension $n$. Here $\operatorname{Aut}(P)$ is the group of bundle automorphisms of $P$.

Our main result asserts that the $T$-reduction of $Z / G$ is a sum over the $\operatorname{Aut}(P)$-orbifolds of the theories $\left(\operatorname{Red}_{T} Z\right)^{P}$ :

Theorem 3.3. Let $T$ be a closed oriented $r$-dimensional manifold, $G$ a finite group and $Z: G$-Cob ${ }_{n+r} \longrightarrow 2$ Vect an $n+r$-dimensional extended $G$-equivariant topological field theory. Then we have a canonical isomorphism

$$
\operatorname{Red}_{T} \frac{Z}{G} \cong \bigoplus_{\substack{\text { isomorphism classes }[P] \\ \text { of } G \text {-bundles over } T}} \frac{\left(\operatorname{Red}_{T} Z\right)^{P}}{\operatorname{Aut}(P)}
$$

of n-dimensional extended topological field theories.

From the assumptions, it follows that this is a finite decomposition.

At the heart of the proof lies a compatibility of dimensional reduction and orbifoldization (Theorem 3.2). The right hand side can be interpreted as a categorified integral with respect to groupoid cardinality (Remark 3.4).

We apply and illustrate our results for theories of Dijkgraaf-Witten type [DW90, FQ93, Mor15, which naturally have an orbifold description [SW19, MW20]: Let DW ${ }_{G}^{\omega}$ be the $n+r$-dimensional extended DijkgraafWitten theory for a finite group $G$ and cohomological twist $\omega \in Z^{n+r}(B G ; \mathrm{U}(1))$. Then its reduction on a closed 
oriented $r$-dimensional manifold $T$ is given by

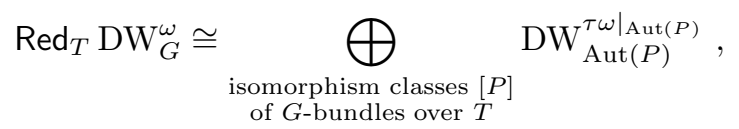

where $\left.\tau \omega\right|_{\operatorname{Aut}(P)}$ is the restriction of the transgression $\tau \omega \in Z^{n}\left(B G^{T} ; \mathrm{U}(1)\right)$ of $\omega$ to the space $B G^{T}$ of maps $T \longrightarrow B G$, see [Wil08] for the concept of transgression. In the case of $T=\mathbb{S}^{1}$ and trivial $\omega$, we obtain

$$
\operatorname{Red}_{\mathbb{S}^{1}} \mathrm{DW}_{G} \cong \bigoplus_{[g] \in G / G} \mathrm{DW}_{\mathrm{C}(g)}
$$

and thereby express the dimensional reduction of the Dijkgraaf-Witten theory for $G$ as a sum over DijkgraafWitten theories for the centralizer subgroups $\mathrm{C}(g)$, where $g$ runs over all conjugacy classes of $G$. This decomposition result was recently conjectured by Qiu-Wang in QW20 for a reduction from dimension $(4,3,2)$ to $(3,2,1)$.

Acknowledgments. We would like to thank Christoph Schweigert for helpful discussions. LM gratefully acknowledges support from the Max-Planck-Institute for Mathematics in Bonn. LW is supported by the RTG 1670 "Mathematics inspired by String theory and Quantum Field Theory" of the DFG.

\section{Equivariant field theories and their orbifolds}

In this section, we provide a brief and informal introduction to extended equivariant field theories and the topological orbifold construction. We try to give the reader a general idea of the concepts involved. For more details, we will give references to the literature. Along the way, we will also present some slight extensions, in particular the technical Lemma 2.5.

\subsection{Equivariant field theories}

An $n$-dimensional topological field theory (depending on the context also referred to as topological quantum field theory) as defined by Atiyah [At88 is a symmetric monoidal functor $Z: \operatorname{Cob}_{n} \longrightarrow$ Vect from the symmetric monoidal category $\mathrm{Cob}_{n}$ of $n$-dimensional bordisms to the symmetric monoidal category Vect of vector spaces (which in this note will always be over the complex numbers). Objects in $\operatorname{Cob}_{n}$ are $n$-1-dimensional closed oriented manifolds $\Sigma$ (we will work with oriented manifolds throughout). A morphism $M: \Sigma_{0} \longrightarrow \Sigma_{1}$ is an equivalence class of compact oriented bordisms from $\Sigma_{0}$ to $\Sigma_{1}$, i.e. an equivalence class of compact oriented $n$-dimensional manifolds $M$ with the choice of an orientation-preserving diffeomorphism $-\Sigma_{0} \sqcup \Sigma_{1} \cong \partial M$, where we denote by $-\Sigma_{0}$ the manifold $\Sigma_{0}$ with the reversed orientation. Composition is given by gluing of manifolds along boundaries.

Over the last decades, this definition has been generalized into various directions. Two of those generalizations will be particularly relevant for us in the sequel:

Equivariant topological field theories. All manifolds appearing in the definition of $\mathrm{Cob}_{n}$ can be compatibly equipped with a map to the classifying space $B G$ of some (finite) group $G$ Tur10-G, TV12, TV14. This leads to a flavor of topological field theory that we will refer to as $G$-equivariant topological field theory.

Extended topological field theories. By taking into account not only manifolds of dimension $n$ and $n-1$, but also $n-2$, one can define a symmetric monoidal bicategory of cobordisms; we refer to [SP09] for its definition and also for the notion of a symmetric monoidal bicategory in general. By slight abuse of notation, we will denote the bicategory of cobordisms by the same symbol $\mathrm{Cob}_{n}$ as its 1-categorical counterpart. For the rest of this note, $\mathrm{Cob}_{n}$ will always denote the $n$-dimensional cobordism bicategory.

The symmetric monoidal category of vector spaces will also be replaced by an appropriate categorification. For our purposes, this will be the symmetric monoidal bicategory 2Vect of Kapranov-Voevodsky 2-vector spaces 
KV94, Mor11. Such a 2-vector space is a complex linear semisimple additive category with finite-dimensional morphism spaces and a finite number of isomorphism classes of simple objects. The 1-morphisms in 2 Vect are linear functors, the 2-morphism are natural transformation. The monoidal product is the Deligne product. Its monoidal unit is the category FinVect of finite-dimensional complex vector spaces. Now one defines an $n$-dimensional extended topological field theory as a symmetric monoidal functor $\operatorname{Cob}_{n} \longrightarrow 2$ Vect (a (symmetric monoidal) functor between (symmetric monoidal) bicategories is here to be understood as a (symmetric monoidal) 2-functor in the weak sense, in particular with plenty of coherence data).

In fact, one can replace $\mathrm{Cob}_{n}$ by an even higher category that takes into account even lower-dimensional manifolds down to the point. This leads to fully extended topological field theories Lur09. In this sense, the extended topological field theories in this note will only be once-extended. One reason for our interest in topological field theories that are once-extended (but not non-extended or further extended) is the deep connection to representation theory (and particularly modular categories) that this type of field theory exhibits in dimension three [RT91, BDSPV15].

In the sequel, we will be interested in topological field theories which are both extended and equivariant as defined and investigated in [SW20]. To this end, one needs to define a symmetric monoidal bicategory $G$-Cob ${ }_{n}$ of $G$-cobordisms for a group $G$. We refer to [SW20, Definition 2.1] for the precise definition. Roughly, $G$-Cob ${ }_{n}$ is defined as follows:

- An object is a closed oriented $n$-2-dimensional manifold $S$ together with a continuous map $\xi: S \longrightarrow B G$.

- A 1-morphism $\left(S_{0}, \xi_{0}\right) \longrightarrow\left(S_{1}, \xi_{1}\right)$ is a cobordism $\Sigma: S_{0} \longrightarrow S_{1}$ between $S_{0}$ and $S_{1}$ equipped with a continuous map $\varphi: \Sigma \longrightarrow B G$ such that the diagram

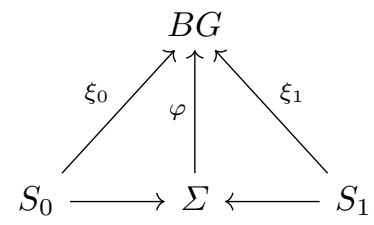

commutes. Composition is defined by gluing manifolds and continuous maps along common boundary components.

- A 2-morphism $\left(\Sigma_{a}, \varphi_{a}\right) \longrightarrow\left(\Sigma_{b}, \varphi_{b}\right)$ between two 1-morphisms $\left(\Sigma_{a}, \varphi_{a}\right)$ and $\left(\Sigma_{b}, \varphi_{b}\right)$ from $\left(S_{0}, \xi_{0}\right)$ to $\left(S_{1}, \xi_{1}\right)$ is an equivalence class of a 2 -cobordism $M: \Sigma_{a} \longrightarrow \Sigma_{b}$, i.e. a certain type of manifold with corners and an identification of $-\Sigma_{a} \sqcup \Sigma_{b}$ with part of its boundary, together with a continuous map $\psi: M \longrightarrow B G$ compatible with $\varphi_{1}$ and $\varphi_{2}$. Horizontal and vertical composition are defined by the gluing of manifolds.

The disjoint union of manifolds induces a symmetric monoidal structure on $G$ - $\operatorname{Cob}_{n}$.

Definition 2.1. An $n$-dimensional extended $G$-equivariant field theory is a symmetric monoidal functor $Z$ : $G$ - $\mathrm{Cob}_{n} \longrightarrow 2$ Vect which has the homotopy invariance property, i.e. it satisfies $Z(M, \psi)=Z\left(M, \psi^{\prime}\right)$ for two 2morphisms $(M, \psi)$ and $\left(M, \psi^{\prime}\right)$ between fixed 1-morphisms if $\psi$ is homotopic to $\psi^{\prime}$ relative boundary. We denote by $G$-TFT $n$ the bicategory of $n$-dimensional extended $G$-equivariant topological field theories. Its morphisms are symmetric monoidal transformations.

In fact, $G$-TFT $n$ is a 2 -groupoid.

Remark 2.2. The direct sum $Z_{1} \oplus Z_{2}$ of equivariant field theories $Z_{1}, Z_{2}: G$ - $\operatorname{Cob}_{n} \longrightarrow 2$ Vect is again a $G$-equivariant topological field theory that can be defined using the following prescription from DJ94 and Tur10-G, I.1.5.3]: On objects $(S, \xi) \in G$-Cob ${ }_{n}$ such that $S$ is non-empty and connected, we define $\left(Z_{1} \oplus\right.$ $\left.Z_{2}\right)(S, \xi):=Z_{1}(S, \xi) \oplus Z_{2}(S, \xi)$. For the empty set, we set $Z_{1} \oplus Z_{2}(\emptyset):=$ FinVect (this is needed for $Z_{1} \oplus Z_{2}$ to be symmetric monoidal). On non-connected manifolds, $Z_{1} \oplus Z_{2}$ is defined as the tensor product of its values on the connected components. Similarly, we define the value of $Z_{1} \oplus Z_{2}$ on connected morphisms as the direct sum of the independent theories (interpreted in an appropriate sense) and extend this definition by tensor products to non-connected morphisms. 
Remark 2.3. In the above definitions, we can replace the space $B G$ with any topological space $T$, see also SW20, which in this context will then be referred to as the target space. We then obtain a symmetric monoidal bicategory $T$ - $\mathrm{Cob}_{n}$ of cobordisms equipped with maps to $T$. A symmetric monoidal functor $T$ - Cob $_{n} \longrightarrow 2$ Vect having the homotopy invariance property will be called an extended homotopy quantum field theory with target space $T$ based on the terminology used in Tur10-G. These theories form again a 2 -groupoid $T$-TFT ${ }_{n}$. If we are given a disjoint union $S \sqcup T$ of spaces $S$ and $T$, the inclusions $S \longrightarrow S \sqcup T$ and $T \longrightarrow S \sqcup T$ induce an equivalence

$$
(S \sqcup T)-\mathrm{TFT}_{n} \stackrel{\simeq}{\longrightarrow} S-\mathrm{TFT}_{n} \times T-\mathrm{TFT}_{n} .
$$

For the proof, we observe $(S \sqcup T)^{X} \cong S^{X} \sqcup T^{X}$ for any connected space $X$, where $Y^{X}$ denotes the space of maps $X \longrightarrow Y$ equipped with the compact-open topology. Using this, we observe that $(S \sqcup T)-\operatorname{Cob}_{n}$ is the coproduct of $S-\mathrm{Cob}_{n}$ and $T-\mathrm{Cob}_{n}$ as symmetric monoidal bicategories, and from this we deduce that (2.1) is an equivalence.

\subsection{The topological orbifold construction}

The main result of [SW20] is a topological orbifold construction for extended equivariant field theories:

Theorem 2.4. For any finite group $G$, there is a canonical functor

$$
-/ G: G-\mathrm{TFT}_{n} \stackrel{-/ G}{\longrightarrow} \mathrm{TFT}_{n}, \quad Z \longmapsto Z / G .
$$

This functor is referred to as the (topological) orbifold construction. The image $Z / G$ of a $G$-equivariant topological field theory $Z$ under this functor is referred to as the orbifold theory of $Z$.

As explained in SW20, the orbifold construction provides a geometric underpinning and generalization of known algebraic orbifoldization procedures such as the orbifoldization of crossed Frobenius algebras Kau02. and categorical orbifoldization Müg04, Kir04, GNN09 including permutation orbifolds Ban98.

We will give a rough description of the orbifold theory $Z / G$ of an extended $n$-dimensional $G$-equivariant topological field theory $Z$ with values in complex 2-vector spaces and refer to SW20 for the details. The orbifold construction is the composition of two constructions which are rather independent:

1. In the first step, we assign to $Z$ an ordinary field theory $\widehat{Z}: \operatorname{Cob}_{n} \longrightarrow 2$ VecBunGrpd with values in the symmetric monoidal bicategory 2 VecBunGrpd of 2 -vector bundles over essentially finite groupoids and their spans as defined in SW18. An object in 2VecBunGrpd is an essentially finite groupoid $\Gamma$ (a groupoid with finitely many isomorphism classes and finite automorphism groups) together with a functor $\varrho: \Gamma \longrightarrow 2$ Vect; we refer to this as a 2 -vector bundle. A morphism from $\left(\Gamma_{0}, \varrho_{0}\right)$ to $\left(\Gamma_{1}, \varrho_{1}\right)$ consists of a span of essentially finite groupoids

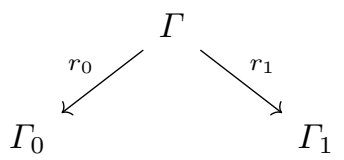

together with a natural transformation $r_{0}^{*} \varrho_{0} \longrightarrow r_{1}^{*} \varrho_{1}$, where $r_{j}^{*} \varrho_{j}$ for $j=0,1$ is the pullback of $\varrho_{j}$ along $r_{j}$ (in the applications that follow, the groupoids will be groupoids of bundles and the legs of the spans will arise from restriction of bundles to the boundaries of a cobordism). Composition is given by forming homotopy pullbacks. The 2-morphisms are equivalence classes of spans of spans of groupoids equipped with modifications encoding the compatibility of the additional structure on the spans.

The field theory $\widehat{Z}$ assigns to an $n$-2-dimensional compact oriented manifold $S$ the fundamental groupoid $\Pi(S, B G):=\Pi\left(B G^{S}\right)$ of the space $B G^{S}$ of maps $S \longrightarrow B G$ equipped with the compact-open topology. An object in this groupoid is a continuous map $S \longrightarrow B G$ and a morphism is a homotopy class of homotopies between maps into $B G$. A homotopy $h$ from $\xi_{0}: S \longrightarrow B G$ to $\xi_{1}: S \longrightarrow B G$ can be seen

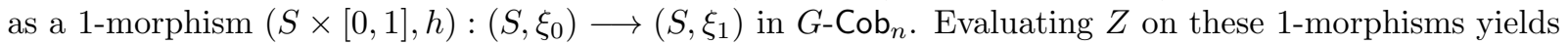


a functor $\Pi(S, B G) \longrightarrow 2$ Vect, i.e. a 2-vector bundle that we define to be $\widehat{Z}(S)$. The topological field theory $\widehat{Z}$ assigns to a 1-morphism $\Sigma: S_{0} \longrightarrow S_{1}$ the span of groupoids

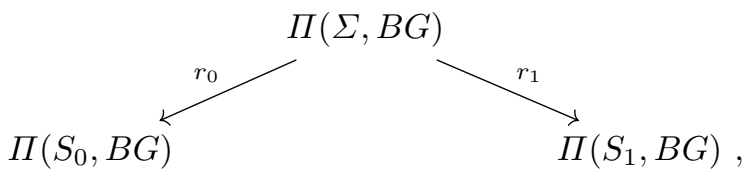

where $r_{0}$ and $r_{1}$ are the obvious restriction functors. Evaluating $Z$ on $\Pi(\Sigma, B G)$ induces the natural transformation which is part of the 1-morphism $\widehat{Z}(\Sigma)$. We refer to the functor

$$
\widehat{-}: G \text {-TFT } \longrightarrow\left[\mathrm{Cob}_{n}, 2 \text { VecBunGrpd }\right]^{\otimes}
$$

as the change to equivariant coefficients. Here $\left[\mathrm{Cob}_{n}, 2 \mathrm{VecBunGrpd}\right]^{\otimes}$ is the 2-groupoid of symmetric monoidal functors $\mathrm{Cob}_{n} \longrightarrow 2 \mathrm{VecBunGrpd}$ (i.e. $2 \mathrm{VecBunGrpd-valued} \mathrm{topological} \mathrm{field} \mathrm{theories).} \mathrm{For} \mathrm{more}$ details and the definition on 2-morphisms, we refer to [SW20].

2. The second ingredient of the orbifold construction is the bicategorical parallel section functor Par : 2 VecBunGrpd $\longrightarrow 2$ Vect constructed in [SW18], see [Tro16] for a 1-categorical analogue. The parallel section functor sends a 2 -vector bundle $\varrho: \Gamma \longrightarrow 2$ Vect to the homotopy limit of $\varrho$, i.e. the 2-vector space of homotopy fixed points. The value of Par on 1-morphisms and 2-morphisms is constructed by a pull-push procedure for 2-vector bundles over spans of bundle groupoids and the integral with respect to groupoid cardinality.

The orbifold theory is now defined by composing the change to equivariant coefficients with the parallel section functor, i.e. (2.2) factorizes as

$$
G \text {-TFT } \stackrel{\widehat{=}}{\longrightarrow}\left[\mathrm{Cob}_{n}, 2 \text { VecBunGrpd }\right]^{\otimes} \stackrel{\text { Paro- }}{\longrightarrow} \mathrm{TFT}_{n} .
$$

It is clear that the orbifold construction with respect to a finite group $G$ (and all its ingredients) can be generalized to an orbifold construction

$$
\Gamma-\mathrm{TFT}_{n} \stackrel{-/ \Gamma}{\longrightarrow} \mathrm{TFT}_{n}, \quad Z \longmapsto Z / \Gamma
$$

with respect to an essentially finite groupoid $\Gamma$. Here we denote by $\Gamma$-TFT ${ }_{n}$ the 2 -groupoid of extended homotopy quantum field theories whose target space is the classifying space of the groupoid $\Gamma$ (Remark 2.3 ). The following Lemma says that the orbifold construction is compatible with the disjoint union of essentially finite groupoids:

Lemma 2.5. For essentially finite groupoids $\Gamma$ and $\Omega$, the square

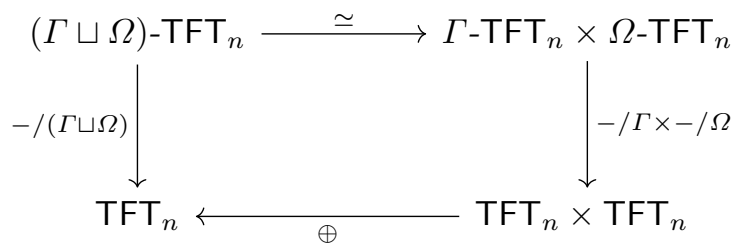

commutes, where the upper horizontal equivalence is from (2.1), the vertical arrows are the topological orbifoldizations with respect to $\Gamma, \Omega$ and $\Gamma \sqcup \Omega$, and the lower horizontal arrow is the direct sum operation from Remark 2.2.

By the definition of the orbifold construction as the composition (2.3), the proof amounts to a compatibility of the direct sum with the change to equivariant coefficients and the parallel section functor which can be verified by a direct computation.

As a consequence of Lemma 2.5, the orbifold with respect to a possibly non-connected essentially finite groupoid can be expressed as a sum over orbifolds with respect to its connected components and hence as a sum over 'ordinary' orbifold theories with respect to a finite group. 


\section{Dimensional reduction of equivariant topological field theories}

Dimensional reduction is the process of producing $n$-dimensional field theories from $n+r$-dimensional ones by evaluating them on manifolds of the form $M \times T$, where $T$ is a fixed closed (oriented) manifold of dimension $r$.

In the mathematical framework of topological field theories this can be realized by pulling back a topological field theory $E: \operatorname{Cob}_{n+r} \longrightarrow 2$ Vect along the symmetric monoidal functor $-\times T: \operatorname{Cob}_{n} \longrightarrow \operatorname{Cob}_{n+r}$ for an $r$-dimensional closed oriented manifold $T$.

This construction does not directly generalize to equivariant field theories $Z$ : $G$ - $\operatorname{Cob}_{n+r} \longrightarrow 2$ Vect since there are multiple ways to construct a continuous map $M \times T \longrightarrow B G$ even after a map $M \longrightarrow B G$ is fixed. One possible way to construct such a map is to use the projection onto $M$. These maps would consequently be constant in the direction of $T$. However, we will instead consider all possible ways to equip $M \times T$ with a G-bundle.

In order to make this precise, first observe that there is a symmetric monoidal functor

$$
-\times T: B G^{T}-\operatorname{Cob}_{n} \longrightarrow G-\operatorname{Cob}_{n+r}
$$

sending an object in $B G^{T}$ - $\operatorname{Cob}_{n}$, i.e. an $n$-2-dimensional closed oriented manifold $S$ equipped with a continuous map $\xi: S \longrightarrow B G^{T}$ to the manifold $S \times T$ equipped with the adjoint map

$$
S \times T \longrightarrow B G, \quad s \times t \longmapsto \xi(s)(t) .
$$

Note that this just uses the standard adjunction $-\times T \dashv-{ }^{T}$. The definition on 1-morphisms and 2-morphisms is accomplished completely analogously.

Definition 3.1. Let $Z: G$-Cob $n+r \longrightarrow 2$ Vect be an $n+r$-dimensional extended equivariant field theory and $T$ a closed oriented $r$-dimensional manifold. The $T$-reduction $\operatorname{Red}_{T} Z: B G^{T}-\operatorname{Cob}_{n} \longrightarrow 2$ Vect of $Z$ is defined as the precomposition

$$
\operatorname{Red}_{T} Z: B G^{T}-\operatorname{Cob}_{n} \stackrel{-\times T}{\longrightarrow} G-\operatorname{Cob}_{n+r} \stackrel{Z}{\longrightarrow} 2 \text { Vect }
$$

of $Z$ with the functor $-\times T$ from (3.1). The dimensional reduction on $T$ takes the form of a functor

$$
\operatorname{Red}_{T}: G-\mathrm{TFT}_{n+r} \longrightarrow B G^{T}-\mathrm{TFT}_{n} .
$$

Our main result is the compatibility of the orbifold construction with dimensional reduction:

Theorem 3.2. Let $T$ be a closed oriented $r$-dimensional manifold and $G$ a finite group. Then the diagram

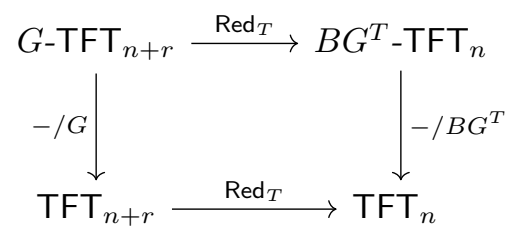

commutes up to natural isomorphism.

Note here that the orbifoldization with respect to $B G^{T}$ (see (2.4) ) makes sense because the space $B G^{T}$ can be seen as the classifying space of an essentially finite groupoid, see [SW20, Lemma 2.8]. 
Proof. By construction of the orbifoldization procedure, it suffices to prove that the two squares

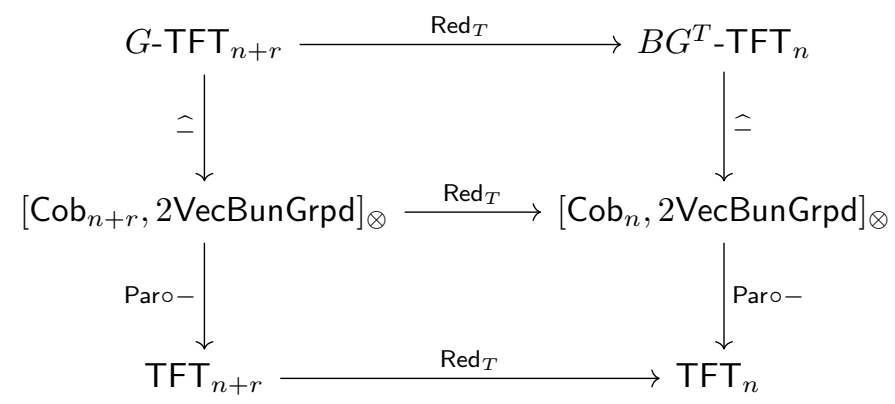

(in which the vertical compositions are (by definition) the orbifoldizations with respect to $G$ and $B G^{T}$, respectively) can be filled with a natural isomorphism. For the lower square, this is obvious because the precomposition and postcomposition with functors commute. Hence, it remains to prove that the upper square commutes up to natural isomorphism.

In order to construct the component of the natural isomorphism at an object $Z: G$ - $\operatorname{Cob}_{n+r} \longrightarrow 2$ Vect in $G-\mathrm{TFT}_{n+r}$ recall that to an $n-2$-dimensional closed oriented manifold $S$ the 2VecBunGrpd-valued topological

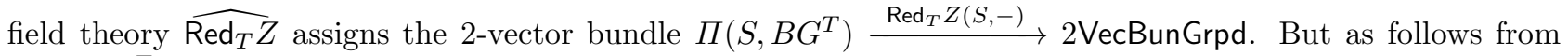
$\Pi\left(S, B G^{T}\right) \cong \Pi(S \times T, B G)$ and the definition of $\operatorname{Red}_{T}$, this 2-vector bundle is canonically isomorphic (in 2 VecBunGrpd $)$ to the 2 -vector bundle $\Pi(S \times T, B G) \stackrel{Z(S \times T,-)}{\longrightarrow} 2$ VecBunGrpd. These isomorphisms extend analogously to 1 -morphisms and 2-morphisms and yield a canonical isomorphism $\widehat{\operatorname{Red}_{T} Z} \cong \operatorname{Red}_{T} \widehat{Z}$. These isomorphisms assemble the natural isomorphism that we need to fill the upper square.

For any $n+r$-dimensional extended $G$-equivariant topological field theory $Z: G$-Cob ${ }_{n+r} \longrightarrow 2$ Vect, we obtain by the above result a canonical isomorphism

$$
\operatorname{Red}_{T} \frac{Z}{G} \cong \frac{\operatorname{Red}_{T} Z}{B G^{T}}
$$

By [SW20, Lemma 2.8] we have $B G^{T} \simeq B \operatorname{PBun}_{G}(T) \simeq \bigsqcup_{[P] \in \pi_{0}\left(\operatorname{PBun}_{G}(T)\right)} B \operatorname{Aut}(P)$, where $\operatorname{PBun}_{G}(T)$ is the

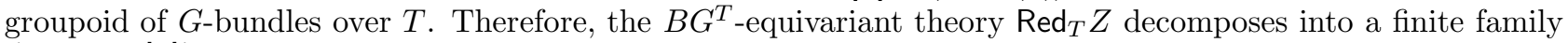
$\left\{\left(\operatorname{Red}_{T} Z\right)^{[P]}\right\}_{[P] \in \pi_{0}\left(\operatorname{PBun}_{G}(T)\right)}$ of Aut $(P)$-equivariant field theories under the equivalence (2.1). We can use Lemma 2.5 to express the right hand side of (3.2) in terms of the orbifold theories of the members of this family and hence arrive at the following decomposition:

Theorem 3.3. Let $T$ be a closed oriented $r$-dimensional manifold, $G$ a finite group and $Z: G$-Cob $n+r \longrightarrow 2$ Vect an $n+r$-dimensional extended $G$-equivariant topological field theory. Then we have a canonical isomorphism

$$
\operatorname{Red}_{T} \frac{Z}{G} \cong \bigoplus_{[P] \in \pi_{0}\left(\operatorname{PBun}_{G}(T)\right)} \frac{\left(\operatorname{Red}_{T} Z\right)^{P}}{\operatorname{Aut}(P)}
$$

of $n$-dimensional extended topological field theories.

For $T=\mathbb{S}^{1}$ (one of the most common choices for dimensional reductions), the bundle groupoid $\mathrm{PBun}_{G}\left(\mathbb{S}^{1}\right)$ is equivalent to the loop groupoid $G / / G$ of $G$, i.e. the action groupoid for the action of $G$ on itself by conjugation. As a consequence, we find

$$
\operatorname{Red}_{\mathbb{S}^{1}} \frac{Z}{G} \cong \bigoplus_{[g] \in G / G} \frac{\left(\operatorname{Red}_{\mathbb{S}^{1}} Z\right)^{g}}{\mathrm{C}(g)}
$$

where $G / G$ is the set of conjugacy classes of $G$ and $\mathrm{C}(g) \subset G$ is the centralizer group of an element $g \in G$. Since the centralizer of the neutral element is $G$, the dimensional reduction of the orbifold theory $Z / G$ contains the summand $\left(\operatorname{Red}_{\mathbb{S}^{1}} Z\right)^{e} / G$ corresponding to the naïve reduction that just allows $G$-bundles which are trivial in $\mathbb{S}^{1}$-direction. 
Remark 3.4. Let $\Gamma$ be an essentially finite groupoid and $f: \Gamma \longrightarrow \mathbb{C}$ and invariant function on $\Gamma$, i.e. a complex-valued function on the object set such that $f(x) \cong f(y)$ whenever $x \cong y$ for $x, y \in \Gamma$. Then we can define the integral of $f$ with respect to groupoid cardinality by

$$
\int_{\Gamma} f(x) \mathrm{d} x:=\sum_{[x] \in \pi_{0}(\Gamma)} \frac{f(x)}{|\operatorname{Aut}(x)|}
$$

see [BHW10] for more background on this primitive, but surprisingly powerful integration theory.

It is hard to overlook the formal resemblance between this integral and the right hand side of (3.3). In fact, we can interpret the right hand side of (3.3) as a categorified integral over the essentially finite groupoid $\operatorname{PBun}_{G}(T)$, where the 'function' to be integrated is $P \longmapsto\left(\operatorname{Red}_{T} Z\right)^{P}$. This function takes values in field theories. Compared to (3.5), the sum is replaced by a direct sum and the division by the sizes of automorphism groups is replaced by topological orbifoldization.

\section{Application to Dijkgraaf-Witten theories}

For any group $G$, a $d$-cocycle $\omega \in Z^{d}(B G ; \mathrm{U}(1))$ gives rise to a $d$-dimensional $G$-equivariant topological field theory. The non-extended version of this field theory is constructed in [Tur10-G, I.2.1]. The extended version that we will denote by

$$
E_{\omega}: G-\mathrm{Cob}_{d} \longrightarrow 2 \mathrm{Vect}
$$

is constructed in MW20. Its value on a closed oriented $d$-dimensional manifold $M$ equipped with a map $\psi: M \longrightarrow B G$ is the number $\left\langle\psi^{*} \omega, \sigma_{M}\right\rangle \in \mathrm{U}(1)$, where $\sigma_{M}$ is a representative for the fundamental class of $M$ and $\langle-,-\rangle$ is the pairing between cochains and chains. This number is often suggestively written as an integral $\int_{M} \psi^{*} \omega \in \mathrm{U}(1)$. Both in [Tur10-G] and MW20, these field theories can be constructed from cocycles on arbitrary spaces.

In order to describe the dimensional reduction of the field theories (4.1), we briefly recall the concept of transgression. Let $T$ be a $r$-dimensional closed oriented manifold and $\sigma_{T} \in Z_{r}(T)$ a representative of its fundamental class. The transgression of a $d$-cocycle $\omega$ on $B G$ to $B G^{T}$ is the $d-r$ cocycle $\tau \omega$ on $B G^{T}$ given by

$$
(\tau \omega)(s)=\mathrm{ev}^{*} \omega\left(s \times \sigma_{T}\right),
$$

where $s$ is a $d-r$-chain on $B G^{T}$ and ev $: B G^{T} \times T \longrightarrow B G$ is the evaluation map.

Proposition 4.1. Let $E_{\omega}$ be the extended $G$-equivariant topological field theory from (4.1) associated to a cocycle $\omega \in Z^{n+r}(B G ; \mathrm{U}(1))$ and $T$ a closed oriented $r$-dimensional manifold. Then the $T$-reduction $\operatorname{Red}_{T} E_{\omega}$ : $B G^{T}-\operatorname{Cob}_{n} \longrightarrow 2$ Vect is naturally isomorphic to $E_{\tau \omega}$.

Proof. We only present the proof on the level of closed oriented $n$-dimensional manifolds. Similar arguments can be applied to the full theories. Let $M$ be a closed $n$-dimensional manifold equipped with a map $\psi: M \longrightarrow B G^{T}$. Then the value of $\operatorname{Red}_{T} E_{\omega}$ on $(M, \psi)$ is

$$
\int_{M \times T}\left(\operatorname{ev} \circ\left(\psi \times \operatorname{id}_{T}\right)\right)^{*} \omega=\left\langle\mathrm{ev}^{*} \omega, \psi_{*} \sigma_{M} \times \sigma_{T}\right\rangle=\left\langle\tau \omega, \psi_{*} \sigma_{M}\right\rangle=\int_{M} \psi^{*}(\tau \omega),
$$

where $\sigma_{M}$ and $\sigma_{T}$ is a representative for the fundamental class of $M$ and $T$, respectively.

The singular cohomology of an aspherical space can be identified with the groupoid cohomology of its fundamental groupoid. We refer to Wil08] for a discussion and concrete formulae for the transgression to loop groupoids.

Dijkgraaf-Witten theories DW90, FQ93, Mor15] are topological field theories which describe gauge theories with finite structure group $G$. The twisted $n$-dimensional Dijkgraaf-Witten theory that we denote by

$$
\mathrm{DW}_{G}^{\omega}: \mathrm{Cob}_{n} \longrightarrow 2 \mathrm{Vect}
$$


depends on the choice of a topological action functional which can be described by a $\mathrm{U}(1)$-valued $n$-cocycle $\omega$ on $B G$. The relation between Dijkgraaf-Witten theories and the content of this note is the fact that they can be written as an orbifold theory,

$$
\mathrm{DW}_{G}^{\omega} \cong \frac{E_{\omega}}{G},
$$

see [SW19, Remark 4.5 (1)] for the statement on the level of non-extended field theories and [MW20, Section 5] for the extended case. From Theorem 3.3 . Proposition 4.1 and (4.2) we directly conclude:

Theorem 4.2. For any closed oriented $r$-dimensional manifold $T$, any finite group $G$ and any cocycle $\omega \in$ $Z^{n+r}(B G ; \mathrm{U}(1))$, the T-reduction of the $\omega$-twisted Dijkgraaf-Witten theory $\mathrm{DW}_{G}^{\omega}$ is given by

$$
\operatorname{Red}_{T} \mathrm{DW}_{G}^{\omega} \cong \bigoplus_{[P] \in \pi_{0}\left(\operatorname{PBun}_{G}(T)\right)} \mathrm{DW}_{\operatorname{Aut}(P)}^{\left.\tau \omega\right|_{\mathrm{Aut}(P)}}
$$

In the special case $T=\mathbb{S}^{1}$ that was treated in (3.4), we prove a recent conjecture of Qiu-Wang QW20:

Corollary 4.3. The dimensional reduction of the $\omega$-twisted Dijkgraaf-Witten theory $\mathrm{DW}_{G}^{\omega}$ is given by

$$
\operatorname{Red}_{\mathbb{S}^{1}} \mathrm{DW}_{G}^{\omega} \cong \bigoplus_{[g] \in G / G} \mathrm{DW}_{\mathrm{C}(g)}^{\tau \omega \mid c(g)}
$$

The precise conjecture in QW20 concerns the untwisted case and a reduction from dimension $(4,3,2)$ to $(3,2,1)$. The statement, however, holds independently of the dimension and also with cohomological twists.

Remark 4.4. We conclude with a few comments on the relation of our results to Conjecture 1.2 of KTZ20] which says that the center of the $n$-category $n V_{\text {ect }}^{\omega}{ }_{G}^{\omega}$ of $G$-graded $n$-vector spaces twisted by an $n+2$-cocycle $\omega \in Z^{n+2}(B G ; \mathrm{U}(1))$ is given by

$$
Z\left(n \operatorname{Vect}_{G}^{\omega}\right) \cong \bigoplus_{[g] \in G / G} n \operatorname{Rep}^{\left.\tau \omega\right|_{C(g)}}(\mathrm{C}(g))
$$

where $\left.n \operatorname{Rep}^{\tau \omega}\right|_{\mathcal{C}(g)}(\mathrm{C}(g))$ is the category of $\left.\tau \omega\right|_{\mathrm{C}(g)}$-twisted $n$-representations of $\mathrm{C}(g)$.

The fact that the right hand sides of (4.3) and (4.4) have a similar form is not a coincidence. In fact, based on Corollary 4.3, we can suggest a strategy for a topological proof of (4.4): It is expected that the $\omega$-twisted Dijkgraaf-Witten theory for the group $G$ admits an extension to a fully extended field theory with values in some suitable target category. Furthermore, the algebraic object describing this fully extended $n+2$-dimensional field theory (its value at the point) is expected to be $n \operatorname{Vect}_{G}^{\omega}$. One expects that there also exists a dual description of the field theory in terms of the higher category $(n+1) \operatorname{Rep}^{\omega}(G)$. The relation between the two descriptions is by identifying $(n+1) \operatorname{Rep}^{\omega}(G)$ with the higher category of modules over $n \operatorname{Vect}_{G}^{\omega}$. If we now assume that a result analogous to (4.3) can be established for the dimensional reduction of the fully extended DijkgraafWitten theory on $\mathbb{S}^{1}$, we can evaluate both sides on the point. On the left hand side, we would find the value of the fully extended Dijkgraaf-Witten on the circle, which by general principles in topological field theories would be the center $Z\left(n \operatorname{Vect}_{G}^{\omega}\right)$. The right hand side would be the right hand side of (4.4) by the mentioned description of Dijkgraaf-Witten theories in terms of twisted representations. Making this argument or even any of its ingredients precise is far beyond the scope of this short note.

\section{References}

[At88] M. F. Atiyah. Topological quantum field theory. Publ. Math. IHÉS, 68:175-186, 1988.

[BHW10] J. C. Baez, A. E. Hoffnung, C. D. Walker. Higher dimensional algebra. VII: Groupoidification. Theory Appl. Categ. 24(18):489-553, 2010.

[Ban98] P. Bantay. Characters and modular properties of permutation orbifolds. Phys. Lett. B 419:175-178, 1998. 
[BDSPV15] B. Bartlett, C. L. Douglas, C. J. Schommer-Pries, J. Vicary. Modular Categories as Representations of the 3-dimensional Bordism Category. 2015. arXiv:1509.06811 [math.AT]

[DW90] R. Dijkgraaf, E. Witten. Topological gauge theories and group cohomology. Comm. Math. Phys. 129(2):393-429, 1990.

[DJ94] B. Durhuus and T. Jónsson. Classification and construction of unitary topological field theories in two dimensions. J. Math. Phys. 35(10):5306-5313, 1994.

[GNN09] S. Gelaki, D. Naidu, D. Nikshych. Centers of Graded Fusion Categories. Algebra \& Number Theory 3(8): 959-990, 2009.

[FQ93] D. S. Freed, F. Quinn. Chern-Simons theory with finite gauge group. Comm. Math. Phys. 156(3):435-472, 1993.

[KV94] M. Kapranov, V. Voevodsky. Braided monoidal 2-categories and Manin-Schechtman higher braid groups. J. Pure Appl. Algebra 92:241-267, 1994.

[Kap10] A. Kapustin. Topological Field Theory, Higher Categories, and Their Applications. Proceedings of the International Congress of Mathematicians Hyderabad, India, 2010.

[Kau02] R. M. Kaufmann. Orbifold Frobenius Algebras, Cobordisms and Monodromies. Contemp. Math. 310:135161, 2002.

[Kir04] A. A. Kirillov. On G-equivariant modular categories. arXiv:math/0401119v1 [math.QA]

[KTZ20] L. Kong, Y. Tian, S. Zhou. The center of monoidal 2-categories in 3+1D Dijkgraaf-Witten Theory. Adv. Math. 360, 2020.

[Lur09] J. Lurie. On the Classification of Topological Field Theories. Current Devel. Math. 2008:129-280, 2008.

[MNS12] J. Maier, T. Nikolaus, C. Schweigert. Equivariant Modular Categories via Dijkgraaf-Witten theory. Adv. Theor. Math. Phys. 16:289-358, 2012.

[Mor11] J. C. Morton. Two-vector spaces and groupoids. Appl. Categ. Structures 19:659-707, 2011.

[Mor15] J. C. Morton. Cohomological Twisting of 2-Linearization and Extended TQFT. J. Homotopy Relat. Struct. 10:127-187, 2015.

[MW20] L. Müller, L. Woike. Parallel Transport of Higher Flat Gerbes as an Extended Homotopy Quantum Field Theory. J. Homotopy Relat. Struct. 15(1):113-142, 2020.

[Müg04] M. Müger. Galois extensions of braided tensor categories and braided crossed G-categories. J. Algebra 277:256-281, 2004.

[QW20] Y. Qiu, Z. Wang. Representations of Motion Groups of Links via Dimension Reduction of TQFTs. arXiv:2002.07642 [math.QA]

[RT91] N. Reshetikhin, V. Turaev. Invariants of 3-manifolds via link polynomials and quantum groups. Invent. Math. 103:547-598, 1991.

[RW18] E. C. Rowell, Z. Wang. Mathematics of Topological Quantum Computing. Bull. Am. Math. Soc. 55(2):183238, 2018.

[SP09] C. J. Schommer-Pries. The classification of two-dimensional extended topological field theories. Ph.D. thesis, 2009. arXiv:1112.1000 [math.AT]

[SW18] C. Schweigert, L. Woike. A Parallel Section Functor for 2-Vector Bundles. Theory Appl. Categ. 33(23):644690, 2018.

[SW19] C. Schweigert, L. Woike. Orbifold Construction for Topological Field Theories. J. Pure Appl. Algebra 223:1167-1192, 2019.

[SW20] C. Schweigert, L. Woike. Extended Homotopy Quantum Field Theories and their Orbifoldization. J. Pure Appl. Algebra 224(1), 2020.

[Tro16] F. Trova. Nakayama categories and groupoid quantization. arXiv:1602.01019 [math.CT]

[Tur10] V. Turaev. Quantum Invariants of Knots and 3-Manifolds. De Gruyter, Berlin 2010.

[Tur10-G] V. Turaev. Homotopy Quantum Field Theory. With appendices by M. Müger and A. Virelizier. European Mathematical Society, 2010.

[TV12] V. Turaev, A. Virelizier. On 3-dimensional homotopy quantum field theory, I. Int. J. Math. 23(9):1-28, 2012.

[TV14] V. Turaev, A. Virelizier. On 3-dimensional homotopy quantum field theory II: The surgery approach. Int. J. Math. 25(4):1-66, 2014. 
[TV92] V. Turaev and O. Viro. State sum invariants of 3-manifolds and quantum 6j symbols. Topology 31(4):865902, 1992.

[Wil08] S. Willerton. The twisted Drinfeld double of a finite group via gerbes and finite groupoids. Algebr. Geom. Topol. 8(3):1419-1457, 2008. 